\title{
Oberwolfach Papers on Mathematics in Undergraduate Study Programs: Challenges for Research
}

\author{
Rolf Biehler $^{1}$ (D) Reinhard Hochmuth ${ }^{2}$
}

Published online: 25 October 2016

(C) Springer International Publishing Switzerland 2016

This editorial introduces the special issue with papers from the conference "Mathematics in Undergraduate Study Programs: Challenges for Research and for the Dialogue between Mathematics and Didactics of Mathematics", which took place at the Oberwolfach Research Institute for Mathematics (MFO Mathematisches Forschungsinstitut Oberwolfach) from Dec 7 to Dec 13 in 2014.

In totality the contributions highlight the diversity of undergraduate students' mathematical experiences among countries as well as the manifold theoretical and empirical foci of research in higher mathematics education. In particular, the latter point indicates that there is a strong need for methodological advances.

The contributions of this special issue represent worked out and extended versions of presentations of the workshop, which was organized by Rolf Biehler (Institut für Mathematik, Universität Paderborn), Reinhard Hochmuth (Institut für Mathematik und ihre Didaktik, Leibniz Universität Hannover), Dame Celia Hoyles (London Knowledge Lab, UCL Institute of Education, University of London) and Patrick W. Thompson (Dept. of Mathematics and Statistics, Arizona State University).

The first two organizers and guest editors of this issue are directors of the German Centre for Higher Mathematics Education (khdm: Kompetenzzentrum Hochschuldidaktik Mathematik) (www.khdm.de/en/), which took the initiative in

Rolf Biehler

biehler@math.upb.de

Reinhard Hochmuth

hochmuth@idmp.uni-hannover.de

1 Institute for Mathematics, Universität Paderborn, Warburger Str. 100, 33098 Paderborn, Germany

2 Institut für Didaktik der Mathematik und Physik, Leibniz Universität Hannover, Welfengarten 1, 30167 Hannover, Germany 
submitting a proposal to the MFO directors as part of their international networking activities.

The Oberwolfach workshop focused on mathematics education for mathematics, engineering and economy majors and for future mathematics teachers. Its aim was to present and discuss these topics with a clear focus on grounding approaches in theoretical and empirical aspects and thereby contributing to overcoming the state of just exchanging opinions and beliefs about undergraduate mathematics education. We saw the conference as an important momentum in creating and sustaining a national and international community of mathematicians and researchers in mathematics education who are interested in tertiary level mathematics education. The abstracts of the invited papers and a summary of the conference's aims were published in Biehler et al. (2014).

After the conference, the editors of IJRUME kindly offered to publish selected papers from the Oberwolfach meeting. A call for contributions was sent to all Oberwolfach participants to submit a paper and all papers underwent the usual reviewing process for IJRUME. The current issue contains 8 papers, the ninth paper by Viviane Durand-Guerrier was mistakenly published in 2015 in IJRUME issue 3, Volume 2. (http://link.springer.com/article/10.1007/s40753-016-0033-2).

The contributions cover a wide range of topics and methods from undergraduate mathematics education, but we can identify four broad categories:

- Research on students' understanding of specific mathematical topics

- Research on the development of students' beliefs and competences and relevant influencing factors within certain courses

- Research on teachers and teaching

- Theoretical approaches for conceptualizing mathematical thinking processes

\section{Research on students' understanding of specific mathematical topics}

The following papers belong to this category: Gravesen, Grønbæk and Winsløw on Task design for students' work with basic theory in analysis: The cases of multidimensional differentiability and curve integrals, the paper by Durand-Guerrier on Conceptualization of the continuum, an educational challenge for undergraduate students and the paper by Roh and Lee on Designing tasks of introductory real analysis to bridge a gap between students' intuition and mathematical rigor: the case of the convergence of a sequence.

All papers in this grouping are concerned with the transition of knowledge to a higher mathematical or formal level: the transition from calculus to analysis, the transition from a school mathematical understanding of real numbers to an understanding at university level, and the transition from an intuitive understanding to a more rigorous understanding of mathematical concepts.

The papers also share a certain research approach

- Developing an understanding for the specific difficulties of students

- Creating (e.g., mathematical) ideas for overcoming the obstacle 
- Implementing specific activities, didactical situations or tasks for promoting students' understanding

- Studying empirically how students worked on the activities and tasks

Whereas Gravesen et al. and Durand-Guerrier share an institutional approach, Roh et al. approach their topic from a cognitivistic point of view.

Gravesen, Grønbæk and Winsløw focus on the transition from calculus to Analysis courses. Against the background of the theory of didactical situations the authors developed tasks for students covering the topics of multivariate differentiation and integration. The developing is accompanied by reflecting on a series of intertwined notions and related questions in view of the potential that the tasks have for facilitating students learning: adidactical potential, linkage potential, research potential and deepening potential, where the latter is further differentiated in terms of ten student activities.

Their research questions can be summarized as follows:

(1) How do the potentials described above function as design guidelines to produce exercises that could improve students' experience and learning in the context of a first course on analysis, focusing on the foundations of calculus in one and several variables?

(2) What realizations of the four potentials are observed in practice?

(3) What major explanations can be given for any differences among theoretical and observed values?

The a priori analysis of two tasks is followed by a posteriori analysis concerning the activities and learning of students in class in view of the tasks. It became clear that differences between the identified potentials and the observed student activities could only partly be attributed to the tasks themselves, but instead are related to norms, attitudes and institutional aspects of the learning setting. These arguments are related to the research presented in Jablonka et al. (this issue). Besides the concrete observations and their analysis, another major progress of the paper is the systematic framework for developing and analyzing tasks as well as subject specific aspects of students' activities. The framework could principally be applied to any thematic field and learning situation.

Durand-Guerrier's paper is based on a theoretical analysis of the problems school and undergraduate students have with the notion of the continuum. Intuitive ideas and conceptualizations of the number line, the dichotomy of discrete and continuous, the concepts of completeness and density-in-itself are in the centre of the problem. The paper provides empirical evidence about the fragmented knowledge students have at the transition between secondary and tertiary level. Based on the theory of didactical situations and an epistemologically oriented a priori analysis of real numbers and the continuum, a didactical milieu with a challenging task for students is created. Students work on a fixed-point problem of a function with various domains: the finite decimal numbers, the rational numbers and the real numbers. The hypothesis that students will construct more adequate conceptions of real numbers when working on and solving the task is supported by the collected empirical data. 
Roh and Lee consider the issue of limits of real sequences in their paper. The mathematical precise definition of limits is a rather well-known obstacle for students learning in the transition from calculus to analysis. The main theoretical background is formed by Fischbein's concept of primary and secondary intuition. The learning environment is built on the basis of an instructional innovation that makes use of what the authors refer to as the $\varepsilon$-strip.

The authors work on the following research questions:

(1) Through the $\varepsilon$-strip activity, how do students develop a new intuition, called secondary intuition, about convergence?

(2) How do students' secondary intuition, developed through the $\varepsilon$-strip activity, play a role in understanding the $\varepsilon-\mathrm{N}$ definition?

(3) How do students' secondary intuition, developed through the $\varepsilon$-strip activity, play a role in proving a theorem related to convergence?

The main body of the article presents a detailed analysis of a sequence of welldeveloped tasks and identifies related phases in developing successively more adequate activities and deeper understanding of regarding the notion of limit. Besides the opportunity to take over the proposed $\varepsilon$-strip activities in one's own courses, the paper provides helpful insight into the cognitive problems students have with the formal limit definition and its development

\section{Research on the development of students' beliefs and competences and relevant influencing factors within certain courses}

The paper by Jablonka, Ashjari and Bergsten on Much palaver about greater than zero and such stuff - First year engineering students' recognition of university mathematics, the paper by Laging and Vosskamp on Determinants of maths performance offirst-year business administration and economics students, and the paper by Greefrath, Koepf, and Neugebauer on Is there a link between preparatory course attendance and academic success? A case study of degree programmes in electrical engineering and computer science belong to this section.

All three papers share the concern with mathematics as a service subject. The paper by Jablonka, Ashjari and Bergsten is a qualitative observational study based on indepth interviews to find out how students see the secondary-tertiary transition and how they interpret the new kind of mathematics at the university level. The paper is not related to any intervention per se but provides valuable information about students' beliefs that offer valuable knowledge for future intervention studies.

The other two papers are interested in how academic success / mathematical performance in a course is related to student variables when they start university (mathematical competence, school grades, beliefs, etc.) and how students' use support systems in or before the first semester mathematics course, such as bridging courses or other support measures, and information on students' learning behavior (e.g., engagement and learning strategies). Both studies use multivariate statistical models and methods to identify important factors and their relative 
influence on mathematical achievement. The design does not allow strong causal inferences but provides valuable indications.

Jablonka, Ashjari and Bergsten implemented a qualitative research concerning the questions whether and how undergraduate students are aware of and reflect the change in the criteria for what counts as legitimate mathematical activity. A great body of interview data and their analysis is complemented by data concerning achieved grades and interviews by lecturers. The theoretical background is built by Bernstein's conceptualizations of pedagogic discourse, elements of Halliday and Hasan's social semiotics, and Eco's idea of the model reader. A further basis for analyzing and discussing the bulk of empirical data is a detailed and systematic compilation of dimensions that characterize the main issues in the secondary-tertiary transition. The authors drew a detailed picture of the students' view regarding changes in levels of rigor, abstraction and formalization that could be rather informative for mathematicians teaching undergraduate students. The analytic framework that integrates the several indicated theoretical approaches is another major contribution of this paper.

The paper by Laging and Vosskamp makes an attempt to determine the predictors of mathematical skills of first-year Business \& Economics students (B\&E). A model of educational production that is used for predicting school achievements is adapted and used as the theoretical basis for the analysis. As predictors, they chose numerous variables which represent study-specific, socio-economic and biographical, motivational and cognitive aspects as well as variables that reflect the learning behavior, working habits and the use of voluntary support (e.g., tutorials). Data from two skills tests and two surveys that were carried out at the University of Kassel in the winter semester 2011/12 and regression techniques are used to identify the determinants of mathematics performance. Based on data from skills tests and questionnaires, regression results showed that for B\&E students the type of graduation, the final school grade, and mathematics grades in school are very important predictors of mathmematics performance when they start their studies. Also, motivational variables contribute to the explanation of mathematics performance. Other socio-economic and biographical variables do not play a significant role. The same applies to the block of variables representing learning strategies. Moreover, the multivariate regression analysis shows that the mathematics skills when measured at the beginning of the studies representing pre-knowledge significantly influence the mathematics skills at a point of measurement nine weeks later after the beginning of their university studies.

Greefrath and Koepf also use a multivariate statistical approach but focus on the relevance and impact of mathematical bridging or preparatory courses with two variants (classroom and e-learning). The design of the study includes assessing the mathematical knowledge by a mathematics test at the beginning of the study and using the regular written examinations after the first term as an indicator of academic success. They focus on the following research questions:

(1) What is the relation between the results of the mathematics test and participation in a preparatory course or the selected type of preparatory course (classroombased or e-learning)?

(2) What is the link between the exam results in the subject of mathematics in the first year of study and participation in a preparatory course or the selected type of preparatory course (classroom-based or e-learning)? 
(3) Does a test at the start of the degree program provide a prediction for the course progress in mathematics in the first semesters of a degree program?

In their analyses they also identify the preparation at school level (basic or advanced course) and the study program taken (electric engineering or computer science) as an influential factor for academic success. The authors provide indications that attending a preparatory course has positive short term effects, but clear long-term effects on academic success were not found.

\section{Research on teachers and teaching}

The research papers adopting a qualitative approach of Jaworski, Mali and Petropoulou on Critical theorizing from studies of undergraduate mathematics teaching for students' meaning making in mathematics and of Gueudet on University teachers' resources systems and documents belong to this section.

The paper by Jaworski, Mali and Petropoulou summarizes and reflects on a series of qualitative research projects that focus on teaching practices in university courses. The detailed observations result directly from intensive collaborations between teaching mathematicians and researchers in mathematics education. The paper covers lectures and small-group tutorials and focuses on the aim of supporting students in meaning making of mathematics. The main research questions with respect to teaching issues are: How to conceptualize the lecturers' teaching and how do the lecturers think about the students and their learning? How do lecturers use the available resource to facilitate teaching and learning? The authors' qualitative analyses take a sociocultural perspective, which is locally complemented by further theoretical approaches tackling the complexity of data and the field. Especially the last point is critically taken into account and discussed in detail.

The question about the use of resources is the focus of the paper by Gueudet. Mathematics teachers interact with resources of various kinds in their work in and out of class. This practice is analyzed by the documentational approach as a theoretical frame that is elaborated to investigate these interactions and their consequences in terms of teachers' practice and teachers' beliefs in particular. According to this approach, using resources teachers develop documents, encompassing these resources and a scheme of use; they also develop structured document systems and resource systems. The following research questions are the focus of the paper:

(1) What are the features of the resource systems developed by university teachers, and how do these features inform us about the teachers' practices at university?

(2) What are the features of the documents developed by university teachers and how do these features inform us about the teachers' practices at university?

Results are developed by interviewing six university teachers. The study exemplifies the analysis of university teachers' document and resource systems and highlights teachers' practice, including their out of class practice and how it is grounded in their professional beliefs. Practices strongly depend on the context, such as the type of the course and the teacher's type of responsibility. 


\section{Theoretical approaches for conceptualizing mathematical thinking processes}

Although all papers of the special issue more or less deeply reflect the question how to conceptualize mathematical thinking processes, there is only the paper by Harel and Soto, Structural Reasoning, which explicitly focuses on this issue. The methodological oriented paper particularly poses the goal to develop an adequate theoretical framework for analyzing structural reasoning phenomena by offering basis units for data analysis using grounded theory. The main contributions are the presentation of a categorization along five major activities (pattern generalization, reduction of an unfamiliar structure into a familiar one, recognizing and operating with structure in thought, epistemological justification, and reasoning in term of general structures) and their detailed illustration from a large body of empirical data. Finally, the field-based events might serve as initial models for researching structural reasoning phenomena.

In totality the contributions highlight the diversity of undergraduate students' mathematical experiences among countries as well as the manifold theoretical and empirical foci of research in higher mathematics education. In particular, the latter point indicates that there is a strong need for methodological advances. Hopefully this special issue contributes to this issue.

Our thanks go to the authors, the reviewers and last but not least the journal editors and in particular Chris Rasmussen, who has accompanied the development process of this issue from the first beginning in always a constructive and very helpful way. Our special thanks also goes to Pat Thompson, who served as a supporting editor in the first round of the review process.

\section{References}

Biehler, R., Hochmuth, R., Hoyles, C., Thompson, P. W. (2014). Mathematics in Undergraduate Study Programs: Challenges for Research and for the Dialogue between Mathematics and Didactics of Mathematics. Oberwolfach Reports, 11(4), 3103-3175. doi:10.4171/OWR/2014/56 and https://www. mfo.de/document/1450/OWR_2014_56.pdf . 